\title{
A method to visualize transdermal nickel permeation in mouse skin using a nickel allergy patch
}

\author{
Tomoko Sugiyama ${ }^{\mathrm{a}, *}$, Motohiro Uo ${ }^{\mathrm{b}, \mathrm{c}}$, Takahiro Wada ${ }^{\mathrm{b}}$, Toshio Hongo ${ }^{\mathrm{b}}$, Daisuke Omagari ${ }^{\mathrm{d}}$, \\ Kazuo Komiyama ${ }^{\mathrm{d}}$, Masakazu Oikawa ${ }^{\mathrm{e}}$, Mikio Kusama ${ }^{\mathrm{a}}$ and Yoshiyuki Mori ${ }^{\mathrm{a}}$ \\ ${ }^{a}$ Department of Dentistry, Oral and Maxillofacial Surgery, Jichi Medical University, Tochigi, Japan \\ ${ }^{\mathrm{b}}$ Advanced Biomaterials Department, Graduate School of Medical and Dental Sciences, Tokyo \\ Medical and Dental University, Tokyo, Japan \\ ${ }^{\mathrm{c}}$ Department of Materials Science, Graduate School of Engineering, The University of Tokyo, Tokyo, \\ Japan \\ ${ }^{\mathrm{d}}$ Department of Pathology, Nihon University School of Dentistry, Tokyo, Japan \\ ${ }^{\mathrm{e}}$ Development and Support Center, National Institute of Radiological Science, Chiba, Japan
}

Received 6 November 2014

Accepted 15 May 2015

\begin{abstract}
Metal patch test is often used in clinical settings when metal-induced contact dermatitis is suspected. However, the transdermal permeation behavior of metal ions from the patch test remains unclear. Current patch tests using high concentrations of metal salt solutions have some side effects, e.g. acute skin reactions to high concentrations of metal salt. To resolve these, estimating metal ion transdermal permeation is wished. In this study, synchrotron radiation X-ray fluorescence (SR-XRF) and micro-focused particle-induced X-ray emission (micro-PIXE) were used to visualize the time-dependent Ni permeation in mouse skin. The cross-sectional diffusion of $\mathrm{Ni}$ was visualized in a time-dependent manner. Our results indicate that maximum $\mathrm{Ni}$ permeation occurs after $24 \mathrm{~h}$ of patch treatment, and the permeated $\mathrm{Ni}$ content was high in the epidermis and spread into the dermis beyond the basal layer. This method may be useful to determine the appropriate solution concentration and duration of administration for the patch test.
\end{abstract}

Keywords: Metal allergy, skin patch test, synchrotron radiation X-ray fluorescence analysis, particle-induced X-ray emission analysis, elemental distribution imaging

\section{Introduction}

Metal allergy patch tests are widely used to diagnose metal-induced contact dermatitis [1,2]. Current patch tests use high concentrations of metal in aqueous salt solutions and a reservoir sheet that allows the test solution to permeate into the skin and induce a local allergic reaction. Although this test is routinely performed to diagnose metal allergies, false-positive and negative reactions are frequently

\footnotetext{
*Corresponding author: Tomoko Sugiyama, Department of Dentistry, Oral and Maxillofacial Surgery, Jichi Medical University, 3311-1 Yakushiji, Shimotsuke, Tochigi 329-0498, Japan. Tel.: +81-285-58-7390; Fax: +81-285-44-8669; E-mail: tomoko_s@jichi.ac.jp.
} 
observed [3,4]. Acute high doses of metal ions can directly damage skin tissue, thus preventing accurate allergy diagnosis. For example, Fischer et al. reported that skin irritation and false positives can occur when using the patch test with high concentrations $(5 \% \mathrm{w} / \mathrm{v})$ of Ni sulfate [5]. Thus, side effects can be avoided by using the minimum concentration of metal salt solution required to induce a local allergic reaction. To achieve this, a method to estimate metal ion transdermal permeation kinetics is essential. The total Ni ion permeation [6] and the permeation depth have been reported using a tape stripping method $[7,8]$. In those reports, an adhesive tape was applied to the Ni permeated skin under constant pressure and gradually removed. This process was repeated 20 times and the Ni concentration in each peeled stratum corneum was evaluated using inductively coupled plasma mass spectroscopy (ICP-MS). Each tape strips removed one layer of corneocytes, approximately. Then, the depth profile of permeated $\mathrm{Ni}$ in the skin layer was estimated. However, visualization of the cross-sectional distribution of permeated $\mathrm{Ni}$ ions with various patch application periods would allow for a better understanding of permeation behavior. We have applied synchrotron radiation X-ray fluorescence (SR-XRF), and micro-focused particle-induced X-ray emission (micro-PIXE) for the distribution imaging of the trace elements contained in human soft tissues [9-11]. SR-XRF method irradiates the monochromatized synchrotron radiation $\mathrm{X}$-rays to the specimens and the generated characteristic (fluorescence) X-rays are detected. SR-XRF spectrum shows fluorescence X-ray peaks derived from various elements contained in the specimen with a negligible background. Therefore, weak fluorescence X-ray peaks derived from trace elements could be easily detected [9]. X-ray irradiation seldom damages specimens, then, SRXRF is suitable for the non-destructive analysis of trace elements in the biomedical specimens. SR-XRF with micro-focused X-rays provides the micro-area analysis and elemental distribution analysis. MicroPIXE method irradiates the focused proton beams to the specimen and generated characteristic X-rays are detected. The specimen damage by the proton irradiation was quite small, then, the non-destructive analysis also could be carried. The focul size of the proton beams are smaller than the typical focused X-ray used in SR-XRF. Therefore, detailed elemental distribution can be obtained by micro-PIXE. In this study, we applied those highly sensitive elemental analysis method to visualize the time-dependent cross-sectional distribution of $\mathrm{Ni}$ using a patch test applied to the epidermis.

\section{Materials and methods}

C57BL/6 mice (50 weeks old) were used in this study. All animal protocols were approved by the Animal Ethics Review Board of the Dental Hospital of Nihon University School of Dentistry, Tokyo, Japan, and conformed to the guidelines of the National Institutes of Health. The back skin of all mice was depilated under general anesthesia. The test solution $(5 \% \mathrm{w} / \mathrm{v} \mathrm{NiSO} 4$ aq.) and closed patch test sheet (Torii Pharmaceutical, Tokyo, Japan) were applied to the depilated skin. The application period was varied between $30 \mathrm{~min}$ and $48 \mathrm{~h}$, after which the mice were slaughtered. The patch was removed and the back skin was gently wiped. The skin that contacted the patch test was excised and immediately frozen in crushed dry ice. All specimens were cut into $1-\mathrm{cm}$-long sections at $-24^{\circ} \mathrm{C}$. The lower part of the skin specimen was embedded in optimum cutting temperature (OCT) compound (Sakura Finetek, Tokyo, Japan) on the cryostat base, and the upper part of the specimen was cut into sections $(20 \mu \mathrm{m})$ using a cryostat (Leica Microsystems, Wetzlar, Germany). The sectioned specimens were placed on Kapton film (12.5 $\mu \mathrm{m}$; Du Pont-Toray, Tokyo, Japan), dried, and subjected to elemental distribution analyses. Adjacent specimen slices were subjected to hematoxylin and eosin (H-E) staining for histopathological analysis. 
Table 1

Detailed experimental conditions for elemental analysis

\begin{tabular}{|c|c|c|}
\hline \multirow[t]{6}{*}{ SR-XRF analysis } & Facility & $\begin{array}{l}\text { BL-4A (Photon Factory, High Energy Accelerator Research Organization, Tsukuba, } \\
\text { Japan) }\end{array}$ \\
\hline & & The electron storage ring was operated at $2.5 \mathrm{GeV}$ and $450 \mathrm{~mA}$. \\
\hline & & Incident X-ray energy $=12.9 \mathrm{KeV}$ \\
\hline & & Focal size $=20 \mu \mathrm{m}$ using polycapillary optics \\
\hline & & Measurement step $=40 \mu \mathrm{m}(1 \mathrm{sec}$ steps $)$ \\
\hline & Data process & PyMca (ESRF, Ver 4.7.3) \\
\hline \multirow[t]{3}{*}{ Micro-PIXE analysis } & Facility & PASTA (National Institute of Radiological Sciences, Chiba, Japan) \\
\hline & & $\begin{array}{l}\text { Micro-focused proton beam }(3.0 \mathrm{MeV}, 2 \mu \mathrm{m} \text { beam diameter, total irradiation }= \\
200 \mathrm{nC})\end{array}$ \\
\hline & Data process & OMDAQ2007 (Oxford Microbeams Ltd., Version 1.3.71.669) \\
\hline
\end{tabular}

Elemental distribution was analyzed by two different methods, depending on the observation area and resolution. Analysis of the entire specimen was performed using SR-XRF. The micro-focused incident $\mathrm{X}$-ray $(12.9 \mathrm{KeV})$ was irradiated, and the specimen stage was two-dimensionally step-scanned. The fluorescence X-ray was detected at each point, and the elemental distribution images were processed. The high-resolution elemental distribution was analyzed using micro-PIXE analysis, which was performed under irradiation by application of a micro-focused proton beam with raster scanning over the target area of the specimen. The characteristic X-rays were detected, and the elemental distribution image was processed. Detailed conditions of the analyses are shown in Table 1.

\section{Results}

Figure 1 shows the H-E stained histopathological images and the elemental distribution images by SR-XRF from the skin cross sections treated with patches for $24 \mathrm{~h}$. The $\mathrm{S}$ distribution image shows the shape of the specimens. The permeated Ni from the patch was clearly observed in the surface of the skin. The histological images and the Ni distribution images by SR-XRF of the specimens after $30 \mathrm{~min}$ to $48 \mathrm{~h}$ of patch exposure are shown in Fig. 2. All SR-XRF measurements were taken under the same ring current and optical geometry. The fluorescent X-ray intensity from each specimen was comparable, as was the relative content. Figure 3 shows the relative content of $\mathrm{Ni}$ in the skin over time as evaluated by the integrated fluorescence X-ray intensity of $\mathrm{Ni} \mathrm{K} \alpha$ over the entire specimen area (as shown in Fig. 2). The Ni relative content increased in a time-dependent manner, reaching a maximum at $24 \mathrm{~h}$ post-application. The Ni relative content decreased after $48 \mathrm{~h}$.

The detailed elemental distribution images ( $\mathrm{S}, \mathrm{P}$ and $\mathrm{Ni}$ ) from the areas with high $\mathrm{Ni}$ accumulation (black and white squares in Fig. 2), as assessed by micro-PIXE, are shown in Fig. 4. Additionally, H-E stained images from the same area are also shown. Ni permeation was clearly observed 30 min after patch application. Ni content in skin, which corresponded to the characteristic X-ray intensity, was increased in a time-dependent manner, reaching a maximum at $24 \mathrm{~h}$ post-application. Ni content subsequently decreased after $48 \mathrm{~h}$. The permeated Ni content was high in the epidermis, and spread into the dermis beyond the basal layer. The decrease in Ni content after $48 \mathrm{~h}$ is like due to complete permeation of $\mathrm{Ni}$ from the patch test sheet and diffusion and intravascular invasion of the permeated $\mathrm{Ni}$ from the skin. Permeation of high concentrations of Ni into the skin was reported to increase blood flow $[12,13]$. Thus, the removal of $\mathrm{Ni}$ by blood flow may cause the decreased $\mathrm{Ni}$ concentration after $48 \mathrm{~h}$. An in vitro study 


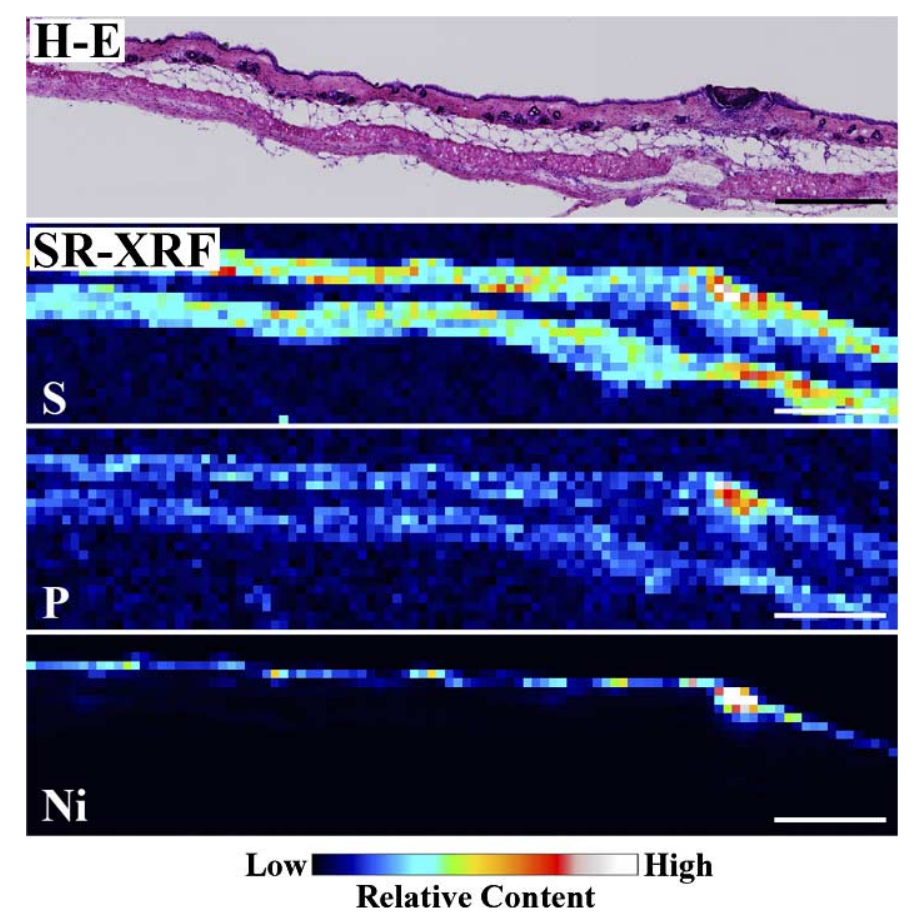

Fig. 1. Histopathological (H-E stained) and elemental distribution images, as obtained by SR-XRF, of mouse skin following Ni allergy patch application for $24 \mathrm{~h}$ (cross section, $\mathrm{bar}=500 \mu \mathrm{m})$. (Colors are visible in the online version of the article; http://dx. doi.org/10.3233/BME-151543.) (Colors are visible in the online version of the article; http://dx.doi.org/10.3233/BME-151543.)

evaluating Ni permeation through the human stratum corneum also reported that the permeation rate of aqueous Ni solutions was highest around $24 \mathrm{~h}$, regardless of the Ni salt species [3,4], in agreement with our results. Current metal allergy tests apply patches for $48 \mathrm{~h}$ and diagnose allergies $48 \mathrm{~h}$ to 1 week after patch application. According to Figs 3 and 4 , the $48 \mathrm{~h}$ application period is sufficient to permeate the $\mathrm{Ni}$ ion; however, most Ni permeation is completed after $24 \mathrm{~h}$. Thus, the patch application period could be shortened. Localization of $\mathrm{P}$ was observed in the same areas as $\mathrm{Ni}$. As shown by the histopathological analysis, $\mathrm{P}$ is derived from inflammatory cells. Thus, the co-localization of $\mathrm{Ni}$ and $\mathrm{P}$ suggests that $\mathrm{Ni}$ permeation from the patch induces local inflammation.

\section{Discussion}

In the present study, we applied SR-XRF and micro-PIXE to visualize permeated $\mathrm{Ni}$ by using a $\mathrm{Ni}$ allergy patch test applied to mouse skin. The skin specimens were freeze-sectioned, and the cross-sectional $\mathrm{Ni}$ distribution was clearly visualized using two different techniques. The distribution of $\mathrm{Ni}$ in the entire specimen could be observed using SR-XRF with lower resolution, and a high-resolution image could be obtained using micro-PIXE within a restricted region of interest in the same specimen. In addition, $\mathrm{H}-\mathrm{E}$ imaging of the adjacent section provided histopathological information. Thus, the feasibility of these analytical methods to visualize metal ion permeation behavior from the metal allergy patch test was demonstrated.

The direct observation method used to assess metal permeation during the patch test revealed the timedependent permeation and cross-sectional distribution of metal ions. This information would be useful 

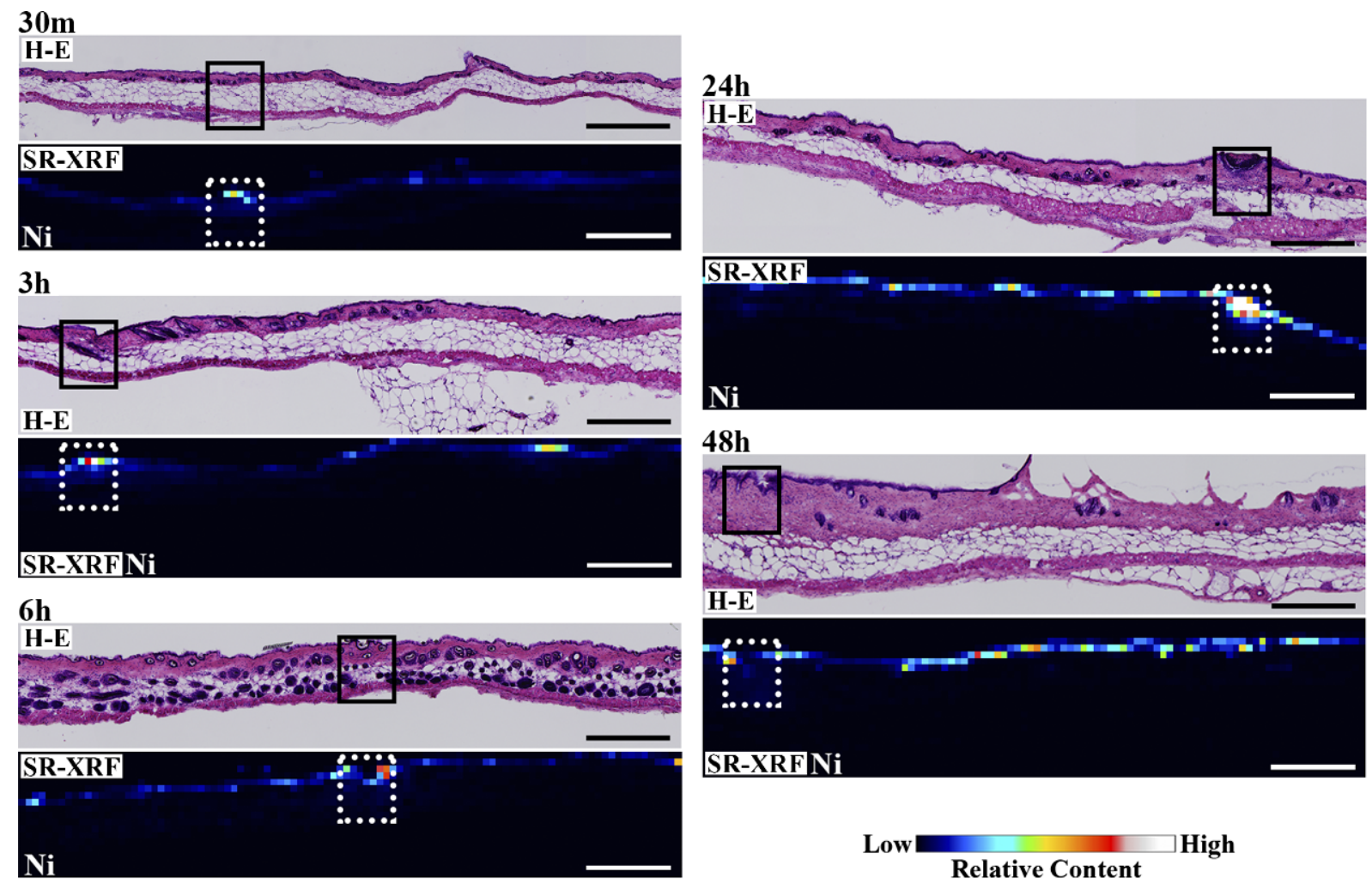

Fig. 2. Histopathological (H-E stained) and elemental distribution images, as obtained by SR-XRF, of mouse skin following $\mathrm{Ni}$ allergy patch application for $30 \mathrm{~min}$ to $48 \mathrm{~h}$ (cross section, bar $=500 \mu \mathrm{m}$ ). (Colors are visible in the online version of the article; http://dx.doi.org/10.3233/BME-151543.)

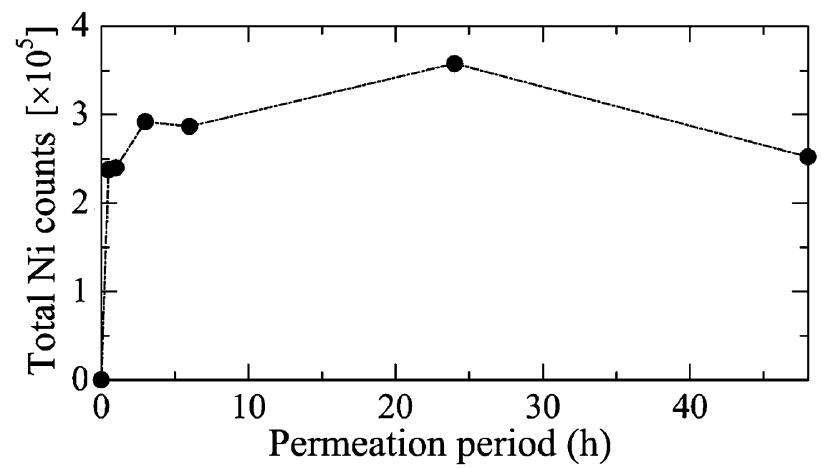

Fig. 3. Time-dependent changes in total permeated Ni within the highly localized areas shown in Fig. 2.

to estimate the optimum concentration for applied metal salt solutions and the application period for the metal allergy patch test. Concerning to the permeation difference of metal ion species, Kubo et al. reported different behavior when using Cr ions in mouse tails [14]. This study showed slow permeation, with the lower layer of the stratum corneum serving as a barrier. While, $\mathrm{Ni}$ was permeated into the dermis beyond the basal layer in this study. Thus, differences in metal ion charge and species may affect mobility. The present method is applicable for all metal ions; thus, the metal ion permeation behavior 


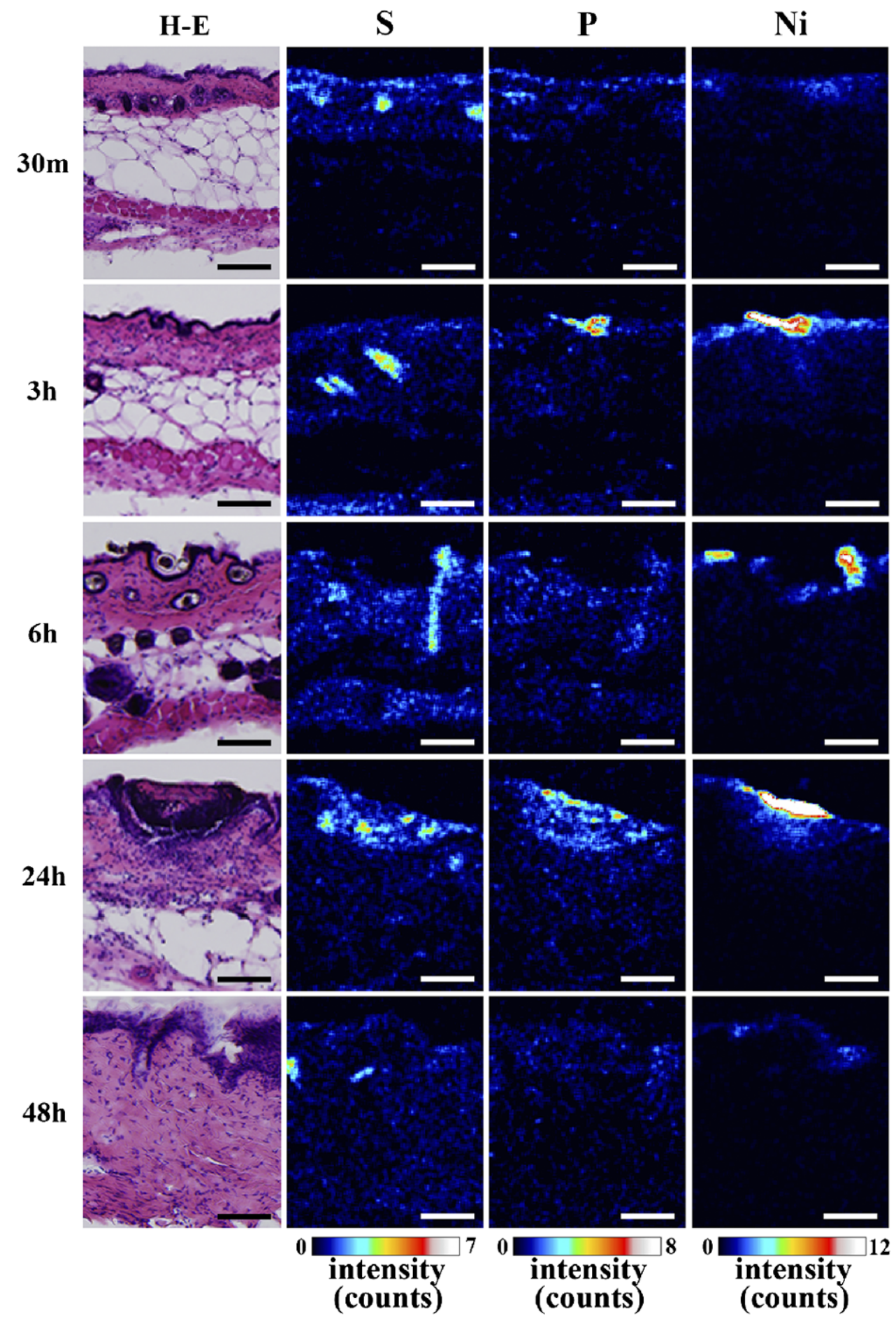

Fig. 4. Histopathological images (H-E stained) and $\mathrm{S}, \mathrm{P}$ and Ni distribution images, as assessed by micro-PIXE, in Ni localized regions $($ bar $=100 \mu \mathrm{m})$. (Colors are visible in the online version of the article; http://dx.doi.org/10.3233/BME-151543.) 
using various patch tests could be estimated and compared. Quantitative analysis should be carried out; however, a method of preparation using low concentration standards has not been established. The quantification of permeated metal ions, including both $\mathrm{Ni}$ and other species, should be carried in future.

\section{Conclusions}

SR-XRF and micro-PIXE were used to analyse Ni allergy patch application in mouse skin. Capillary focused SR-XRF is appropriate to obtain a rapid, wide area scan with low resolution. Micro-PIXE allows for a high resolution analysis within a restricted region of interest. These methods are both nondestructive. As a result, the direct observation of the permeated metal ions during the patch test could be carried out using these methods in combination. The time-dependent permeation and cross-sectional distribution of metal ions were revealed. This information would be useful to estimate the metal ion permeation of the metal allergy patch test.

\section{Acknowledgements}

The SR-XRF measurements were performed with the approval of the Photon Factory Program Advisory Committee (Proposal No. 2014G017). This work was financially supported by the Japan Society for the Promotion of Science KAKENHI (grant No. 23390438 to M. Uo). T. Sugiyama is a supported by a research fellowship for young scientists from the Japan Society for the Promotion of Science.

\section{References}

[1] J. Bourke, I. Coulson and J. English, Guidelines for the management of contact dermatitis, Br. J. Dermatol. 145 (2001), $877-885$.

[2] P.G.M. van der Valk, S.A. Devos and P.J. Coenraads, Evidence-based diagnosis in patch testing, Contact Dermatitis 48 (2003), 121-125.

[3] C.M. Mowad, Patch testing: Pitfalls and performance, Curr. Opin. Allergy Clin. Immunol. 6 (2006), 340-344.

[4] T.J. Diepgen and P.J. Coenraads, Sensitivity, specificity and positive predictive value of patch testing: The more you test, the more you get? ESCD working party on epidemiology, Contact Dermatitis 42 (2000), 315-317.

[5] T. Fischer and I. Rystedt, False-positive, follicular and irritant patch teat reactions to metal salts, Contact Dermatitis 12 (1985), 93-98.

[6] H. Tanojo, J.J. Hostýnek, H.S. Mountford and H.I. Maibach, In vitro permeation of nickel salts through human stratum corneum, Acta Derm. Venereol. Suppl. 212 (2001), 19-23.

[7] J.J. Hostýnek, F. Dreher, A. Pelosi, A. Anigbogu and H.I. Maibach, Human stratum corneum penetration by nickel; in vivo study of depth distribution after occlusive application of the metal as powder, Acta Derm. Venereol. Suppl. 212 (2001), $5-10$.

[8] J.J. Hostýnek, F. Dreher, T. Nakada, D. Schwindt, A. Anigbogu and H.I. Maibach, Human stratum corneum adsorption of nickel salt; Investigation of depth profiles by tape stripping in vivo, Acta Derm. Venereol. Suppl. 212 (2001), 11-18.

[9] M. Uo, T. Wada and T. Sugiyama, Applications of X-ray fluorescence analysis (XRF) to dental and medical specimens, J. Dent. Sci. Rev. 51 (2015), 2-9.

[10] T. Sugiyama, M. Uo, T. Wada, D. Omagari, K. Komiyama, T. Noguchi, Y. Jinbu and M. Kusama, Estimation of trace metal elements in oral mucosa specimens by using SR-XRF, PIXE, and XAFS, Biometals 28 (2015), 11-20.

[11] T. Sugiyama, M. Uo, T. Wada, D. Omagari, K. Komiyama, S. Miyazaki, C. Numako, T. Noguchi, Y. Jinbu, M. Kusama and Y. Mori, Detection of trace metallic elements in oral lichenoid contact lesions using SR-XRF, PIXE, and XAFS, Sci. Rep. 5 (2015), 10672. doi:10.1038/srep10672.

[12] J.E. Wahlberg, Nickel: The search for alternative, optimal and non-irritant patch test preparations: Assessment based on laser Doppler flowmetry, Skin Res. Technol. 2 (1996), 136-141. 
[13] J.E. Wahlberg, Nickel chloride or nickel sulfate? Irritation from patch-test preparations as assessed by laser Doppler flowmetry, Dermatol. Clin. 8 (1990), 41-44.

[14] A. Kubo, I. Ishizaki, A. Kubo, H. Kawasaki, K. Nagao, Y. Ohashi and M. Amagai, The stratum corneum comprises three layers with distinct metal-ion barrier properties, Sci. Rep. 3 (2013), 1731. doi:10.1038/srep01731. 